\title{
Ecological versatility and the assembly of multiple competitors: cautionary notes for assembly inferences
}

\author{
Victor S. Saito, ${ }^{1,2,5}$ Fabien Laroche, ${ }^{3}$ Tadeu Siqueira, ${ }^{4}$ and Sandrine Pavoine ${ }^{2}$ \\ ${ }^{1}$ Departamento de Ciências Ambientais - DCAm and Programa de Pós-Graduação em Ecologia e Recursos, Universidade Federal de São \\ Carlos, Rodovia Washington Luis, km 235, 13565-905 São Carlos, São Paulo, Brazil \\ ${ }^{2}$ Centre d'Ecologie et des Sciences de la Conservation (CESCO UMR7204), Sorbonne Universités, MNHN, CNRS, UPMC, CP51, 43-61 rue \\ Buffon, Paris 75005 France \\ ${ }^{3}$ Irstea, UR EFNO, Nogent-sur-Vernisson F-45290 France \\ ${ }^{4}$ Departamento de Ecologia, Instituto de Biociências, Universidade Estadual Paulista (UNESP), Av. 24-A, 1515, 13506-900, Rio Claro, São \\ Paulo, Brazil
}

\begin{abstract}
The role of niche differences and competition is invoked when one finds coexisting species to be more dissimilar in trait composition than expected at random in community assembly studies. This approach has been questioned as competition has been hypothesized to either lead to communities assembled by similar or dissimilar species, depending on whether species similarity reflects fitness or niche differences, respectively. A current problem is that the arguments used to draw relationships between competition and species similarity are based on pairwise theoretical examples, while in nature competition can occurs among a constellation of species with different levels of versatility in resources used. By versatility we mean the documented ability of some species to escape competition for commonly used resources by changing for marginal and unused resources. Thus, a versatile species will have the ability to decrease niche overlap with all other species when facing strong competitors. When these species are embedded in multiple interactions the role of pairwise niche and fitness differences could be reduced due to indirect effects and thus competition would not be detectable. Here we developed a coexistence theory where competition occurs simultaneously among multiple species with different levels of versatility and then used it in a simulation to unravel patterns of species similarity during community assembly. We found that simulated communities can be assembled by species with more, less or equal similarity compared to a null model when using a mean distance based metric (SES.MPD). However, contrasting these varied results, we consistently found species overdispersion using a nearest neighbor-based metric (SES.MNTD), even when species differences reflected more directly their competitive abilities than their niche differences. Strong tendency to overdispersion emerged when high ecological versatility promoted large niche differences and enabled coexistence. This is because versatility to use marginal resources compensates possible fitness differences among species. Our findings provide mounting evidence of the important role of minimum niche differences and versatility in resource consumption for species embedded in multiple direct and indirect interactions.
\end{abstract}

Key words: assembly rules; clustering; functional diversity; functional versatility; limiting similarity; multispecies competition; niche theory; overdispersion.

\section{INTRODUCTION}

It has become common practice in community ecology to assume that communities composed of more dissimilar species than expected at random (hereafter species overdispersion) are predominantly assembled by interspecific competition (Weiher and Keddy 1995, Webb 2000, HilleRisLambers et al. 2012, D'Andrea and Ostling 2016). This inference is grounded on MacArthur and Levins's (1967) limiting similarity hypothesis, which claims the existence of a limit to the resource use overlap between species for their stable coexistence. Accordingly, communities composed of similar species (hereafter species clustering) are seen as evidence for relaxed competition for resources and strong abiotic filtering selecting phenotypes well adapted to the environment (Weiher and Keddy 1995).

After years of intense application of this approach, Mayfield and Levine (2010) challenged it by explaining

Manuscript received 31 March 2017; revised 28 November 2017; accepted 1 February 2018. Corresponding Editor: Nathan J. B. Kraft.

${ }^{5}$ E-mail: victor.saito@gmail.com that species clustering can also be the result of interspecific competition if species similarities (e.g., calculated using morphologic, behavioral, metabolic traits) reflect their competitive hierarchy (see also HilleRisLambers et al. 2012, Adler et al. 2013, D'Andrea and Ostling 2016, Cadotte and Tucker 2017). This happens because, in contrast to the traditional view, not all species differences should promote coexistence. According to Chesson (2000), species coexistence depends on two kinds of interspecific differences. First, stabilizing niche differences (hereafter niche differences) favor coexistence by reducing the overlap in resource use between species in time or space, making intraspecific competition more intense than interspecific competition (Chesson 1991). Second, specieslevel average fitness differences (hereafter fitness differences) determine which species will outcompete others in absence of niche differences (Chesson 2000). A balance between niche and fitness differences determines stable coexistence and the pairwise coexistence condition is that niche differences must overcome fitness differences (Chesson 2000). So, if species similarities relate to their fitness equalities, then one could expect to find species clustering 
around an optimum trait value when competition rules the assembly (Mayfield and Levine 2010).

Understanding how differences in species morphology, behaviors and strategies influence niche and fitness differences between them is a complex challenge (Godoy et al. 2014, Herben and Goldberg 2014, Kraft et al. 2015, D'Andrea and Ostling 2016). Several traits can impact both niche and fitness differences, even if the net impact on one is higher than on the other (Kraft et al. 2015). For example, two animal species competing for different prey can have distinct preferences for prey size and taxonomic identity of prey. Differences in taxonomic preferences could favor coexistence through resource partitioning but size preferences could lead to competitive asymmetry given that feeding on larger animals may improve species fitness. Thus, differences in taxonomic and size preferences would contribute to both niche and fitness differences. Therefore, it is not clear whether analyzing together traits that drive stabilizing and equalizing mechanisms simultaneously should give clustered, segregated or random patterns.

Beyond the importance of niche and fitness differences for competitive outcomes, two characteristics of species competition are usually overlooked in community assembly studies. First, competition generally happens between several species in nature (Pianka 1974), not only in pairwise situations, as commonly considered in conceptual and experimental examples (Mayfield and Levine 2010, Chu and Adler 2015). The addition of a third (or more) species to a community composed of two species modifies the competitive network. This happens because three species can experience indirect effects of each other (Levine 1976, Lawlor 1979). For example, let us consider species A, B and C: species A has a direct effect on species B by directly competing for resources, but also has an indirect effect by competing with the third species $\mathrm{C}$, which is a common competitor of species $\mathrm{A}$ and $\mathrm{B}-$ thus the full impact of species A on B would be the sum of the direct and indirect effects. Given that, the pairwise coexistence condition - i.e., niche differences must exceed fitness differences (Chesson 2000) - is not the only determinant of species coexistence, as it is also influenced by the other species that compose such a community.

Second, one of the most used models of species competition considers that competition occurs over a linear array of resources and each species have a trait that makes them able to consume a range of this array of resources (the MacArthur's consumer-resource model, MacArthur 1970). In this case, trait differences determine the resource use overlap and thus the interaction strength among species (see

\section{MacArthur's consumer-resource model}

$\mathbf{A}$

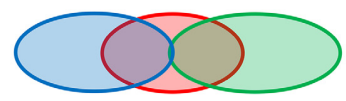

Sp. 1 Sp. 2 Sp. 3
B

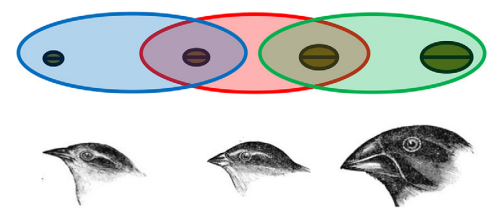

\section{The interaction strength}

Species have one trait that makes them able to capture some resources in the array of resources. As more similar their trait value, the most intense the interaction among them. Empirically, this interaction strength would be inversely proportional to the differences in their trait values.

\section{$\boldsymbol{\alpha}_{i j=1-\mid} \mid$ rait $_{i}$ - trait $_{j} \mid$}

$\boldsymbol{\alpha} i j$ is the interaction strength between species $i$ and $j$. trait $i$ is the trait value of species $i$.

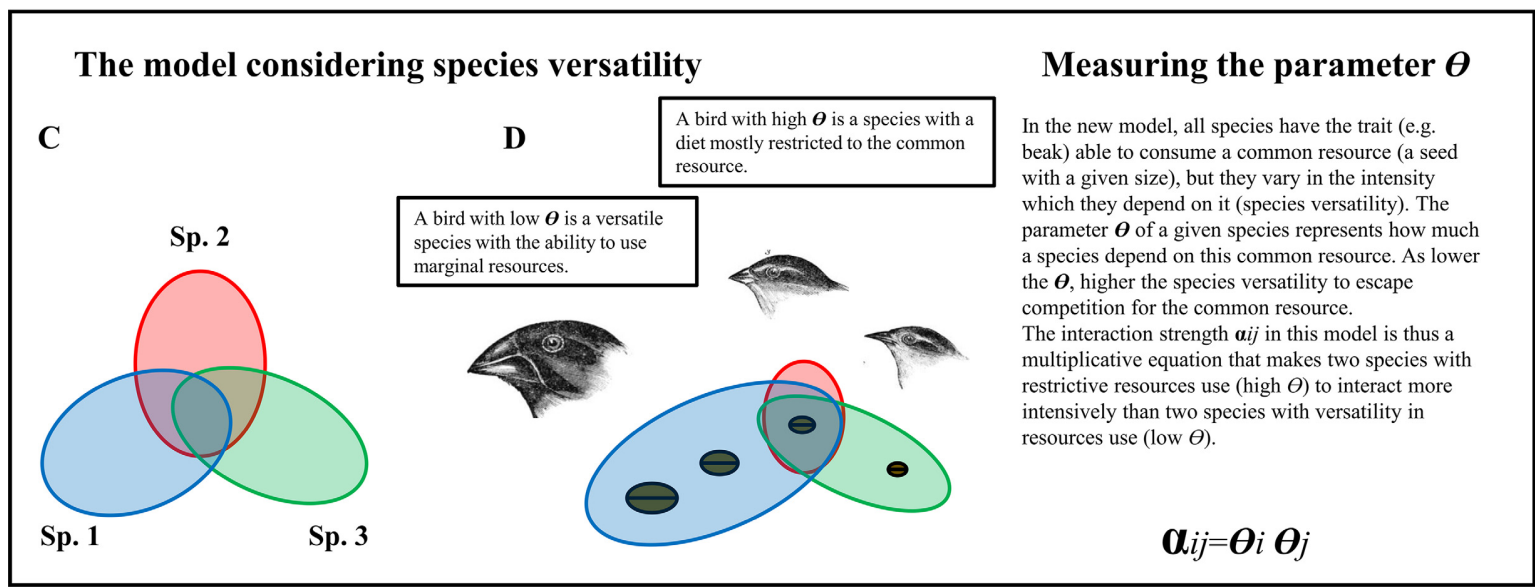

FIG. 1. The differences between MacArthur's consumer-resource model and our new model with ecological versatility. (a) In MacArthur's model, species have trait differences that make their interactions less intense, given that competition happens for a linear array of resources and each species is able to consume part of this array. (b) In nature, this would be the case when, for example, birds have different beak sizes and each size enables the consumption of a range of seed sizes. So, differences in beak sizes always decrease the interaction strength. (c) In our model, species also have traits that enable them to consume part of the array of resources, but all species can consume one common resource. (d) This would be the case when all species of birds can eat a common seed or insect, but not all species are dependent on these items. Some species have a more distinct beak morphology that enables them to also eat other items. In this case, the interaction strength and thus niche overlap are determined by how much a species depends on this common resource. 
Fig. 1). However, competition in nature can happens in a different way. In other documented cases, all species will be able to consume one common resource (in general an abundant resource), but they may vary in the ability to consume other resources, changing their dependency on this common resource when facing competition (Liem 1980, 1990, Bellwood et al. 2006). For example, Liem (1980) found that morphological differentiation in mouth apparatus in cichlids determine the ability of species to escape competition for common preys (by changing for a different resource) in the presence of strong competitors. In this case, differences in traits impacts their versatility and thus could determine niche differences with all other species simultaneously using the coexistence framework (see Fig. 1 for a schematic explanation of the differences between MacArthur's model and our model considering species versatility).

So, based on the potential impact that multispecies competition and species versatility may have on community assembly, we investigated the strength of commonly used metrics of species dispersion (e.g., mean pairwise and nearest neighbor metrics, Weiher and Keddy 1995, Webb 2000) to detect signatures of competition under a mechanistic model of competition. We first developed the underlying theory of competition considering species versatility upon Chesson's (2000) coexistence theory and then used it within a simulation based on sequential colonization from a species pool. We observed the emergence of species clustering, overdispersion and random patterns in simulated communities fueling the ongoing discussion on the use of species dispersion metrics to infer assembly processes.

\section{Methods}

\section{Theory development}

We used a model to simulate community assembly based on modern coexistence theory and under multispecies competition (see Appendix S2 for a detailed version of the model and proofs). We aimed a reference model that respects Chesson's (2000) following to the long-term low-density growth rate of a species $i$ in a multispecies system (Eq. 4 in Chesson 2000):

$$
\bar{r}_{i} \approx b_{i}\left(k_{i}-\frac{\sum_{s \neq i} k_{s}}{n-1}\right)+b_{i} \frac{(1-\rho)}{n-1} D
$$

where $n$ is the number of species in the system, the $k \mathrm{~s}$ are measures of fitness of individual species, $\left(\sum_{s \neq i} k_{s}\right) /(n-1)$ is

the average fitness of the competitors of $i, \rho$ is a measure of niche overlap, and $D$ is a positive constant. This equation is composed of an equalizing term

$$
b_{i}\left(k_{i}-\frac{\sum_{s \neq i} k_{s}}{n-1}\right)
$$

and a stabilizing term

$$
b_{i} \frac{(1-\rho)}{n-1} D
$$

In this kind of community model, a set of species is said to coexist if all individual species can grow from low density in the community made of the $n-1$ other species at equilibrium (i.e., community feasibility).
We started with MacArthur's consumer-resource model where $n$ species compete for $m$ resources (Eqs. 1 and 2 in Chesson 1990):

$$
\left\{\begin{array}{l}
\frac{1}{X_{i}} \frac{d X_{i}}{d t}=b_{i}\left[\sum_{l=1}^{m} c_{i l} w_{l} R_{l}-m_{i}\right] \\
\frac{1}{R_{l}} \frac{d R_{l}}{d t}=r_{l}\left(1-\frac{R_{l}}{K_{l}}\right)-\sum_{i=1}^{n} c_{i l} X_{i}
\end{array}\right.
$$

where $i$ is a consumer species; $X_{i}$ is population density; $R_{l}$ is the density of food resource $l ; w_{l}$ is the value of one unit of resource $l$ to the consumer; $c_{i l}$ is the rate at which consumer species $i$ captures resource $l$ per unit abundance of resource $l ; m_{i}$ is the total value of resource that must be harvested per capita for the growth rate to be exactly $0 ; b_{i}$ is a factor converting the resource excess into the per capita growth rate; $r_{l}$ is the growth rate and $K_{l}$ the carrying capacity for resource $l$.

Assuming a separation of time scales between resource and consumer dynamics yields (see Appendix S2):

$$
\frac{1}{\tilde{X}_{i}} \frac{d \tilde{X}_{i}}{d t}=\tilde{b}_{i}\left[\tilde{k}_{i}-\sum_{j=1}^{n} \tilde{\alpha}_{i j} \tilde{X}_{j}\right]
$$

where $\tilde{X}_{i}$ and $\tilde{b}_{i}$ are rescaled quantities corresponding to $\tilde{X}_{i}$ and $b_{i}$ from equation (4), $\tilde{k}_{i}$ is a rescaled quantity corresponding to $\sum_{l=1}^{m} c_{i l} w_{l} R_{l}-m_{i}$ in equation (4) and $\tilde{\alpha}_{i j}$ is a rescaled interaction coefficient between species $i$ and $j$, comprised between 0 and 1 , which corresponds to a resource overlap between the two species (in particular $\tilde{\alpha}_{i j}$ ).

Consider species $i$ at low density, and assume that the $n-1$ other species can coexist. Then all the other species $j \neq i$ harboring their equilibrium density $\tilde{X}_{j i}$ (second subscript " $i$ " meaning "in the absence of species $i$ "). Then the long-term growth rate at low density of species $i, \bar{r}_{i}$, is obtained by neglecting $\tilde{X}_{i}$ and setting the $\tilde{X}_{j}$ s to $\tilde{X}_{j i} \mathrm{~s}$ in (5):

$$
\bar{r}_{i}=\tilde{b}_{i}\left[\tilde{k}_{i}-\sum_{\substack{j=1 \\ j \neq i}}^{n} \tilde{\alpha}_{i j} \tilde{X}_{j i}\right] .
$$

To derive an equation for the long-term low-density growth rate of a species in the form of Chesson's approximation for multispecies system (Eq. 1), we considered a particular type of interactions among species that reflect species versatility: for all pairs of species $i \neq j$, we assumed that $\tilde{\alpha}_{i j}=\theta_{i} \theta_{j}$, where $\theta=\left(\theta_{1}, \theta_{2}, \ldots, \theta_{n}\right)$ is a vector of positive real numbers between 0 and 1 . In particular, $\tilde{\alpha}_{i i}=1$. Empirically, $\theta$ can be a measure of resource use restriction or versatility (e.g., Bellwood et al. 2006 used the species distance to the centroid of a multidimensional space of species distances as a measure of versatility). High values of $\theta$ means that a species will depend mostly on the common resource all species can consume, while species with low $\theta$ will be those with the versatility to use marginal resources when facing competition (see Fig. 1). 
Importantly, we show in Appendix S2 that any set of interactions of this type can be obtained by adjusting the basic parameters of the model (resource renewal dynamics and consumption vectors), as long as the number of resource types is equal or greater than the number of species. As similar to the MacArthur's model, resource types can be anything that can vary and can be depleted for other species, i.e., seed size ranging from 1 to $100 \mathrm{~cm}$ can be considered 100 resources for birds if each size encompasses a resource that provide niche differences and can be limiting:

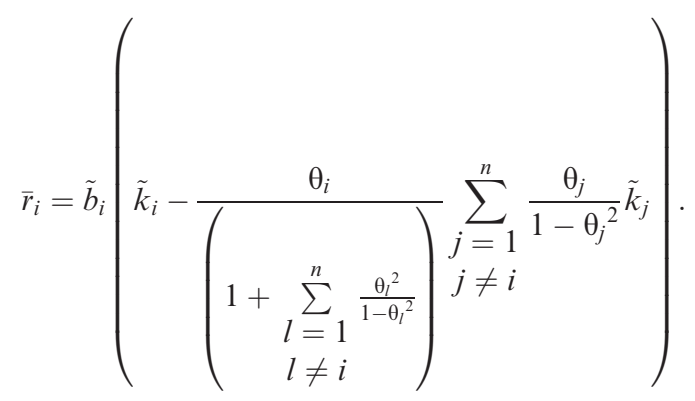

We now seek to decompose equation (7) into stabilizing and equalizing terms as in Chesson's (2000) framework. To find the stabilizing term, we first looked at the long-term growth rate at low density when all the species have the same fitness $\tilde{k}$. In this case, only the stabilizing should act on coexistence. When all species have equal fitness, (7) becomes:

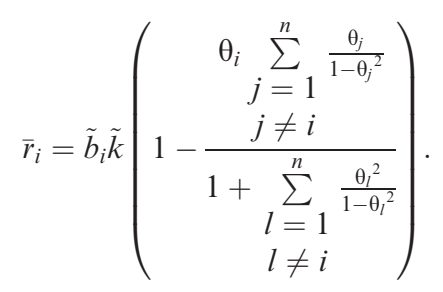

We now search for the corresponding equalizing term in an additive decomposition as in Chesson's (2000) framework (Eq. 1 above). Starting from (7), we obtained (see Appendix S2 for detailed proofs) the following final expression for the long-term growth rate of a species:

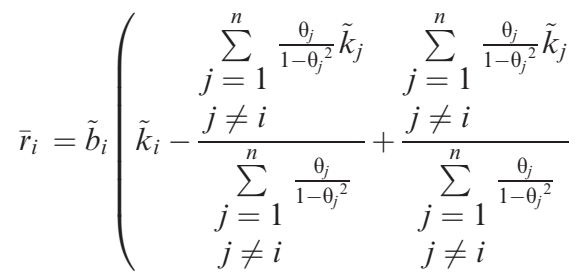

$$
\begin{aligned}
& \left(\begin{array}{c}
\sum_{\substack{j=1 \\
j \neq i}}^{n} \frac{\theta_{i} \theta_{j}}{1-\theta_{j}{ }^{2}} \\
\left.1-\frac{\sum^{2}}{1+\sum_{l=1}^{n} \frac{\theta_{l}{ }^{2}}{1-\theta_{l}{ }^{2}}}\right)
\end{array}\right)=\tilde{b}_{i}\left(\tilde{k}_{i}-\tilde{k}_{i}+\left(1-\rho_{i}\right) \tilde{k}_{i}\right)
\end{aligned}
$$

where

$$
\begin{gathered}
\sum_{i=1}=\frac{\sum_{j=1}^{n} \frac{\theta_{j}}{1-\theta_{j}{ }^{2}} \tilde{k}_{j}}{\sum_{j=1}^{n} \frac{\theta_{j}}{1-\theta_{j}{ }^{2}}} \\
j \neq i \\
\rho_{i}=\frac{\sum_{i} \sum_{j=1}^{n} \frac{\theta_{j}}{1-\theta_{j}{ }^{2}}}{1+\sum_{\substack{j=1 \\
l \neq i}}^{n} \frac{\theta_{l}{ }^{2}}{1-\theta_{l}{ }^{2}}} .
\end{gathered}
$$

We thus retrieved in (9) a stabilizing term with a form consistent with Chesson's (2000) equation (Eq. 1).

The main difference with Chesson's (2000) framework is that $\bar{k}$ is not a simple arithmetic mean of the fitness values but a weighted mean, where weights $\left(\frac{\theta_{j}}{1-\theta_{j}^{2}}\right)$ depend on some interaction effects with other species. Changing interactions among species thus changes the $\bar{k}_{i}$. Our theory provides a multispecies extension of the pairwise Chesson's theory in the case when interactions strength among species simultaneously affects in the same direction the competition with all the other species. This is because all species can consume a common resource, but vary in their versatility to consume other resources (see Fig. 1).

\section{Simulation description}

To simulate community assembly, we created a species pool with 100 species. For each species we settled values of $k_{i}$ and $\theta_{i}$ - the parameters of species in our coexistence model (Eq. 9). The values of these species parameters determine niche and fitness differences and thus both are necessary to determine coexistence in communities using equation (Eq. 9). Biologically, $k_{i}$ would be related to one or several morphological, behavioral or other traits that influence species competitive ability. Theta, however, represents a synthetic measure of ecological versatility indicating the extent to which a species depends on a common resource that all other species can consume (see Fig. 1). We calculated fitness differences and niche differences resulting in two species distance matrix, one that represented species niche overlap $\left(1-\theta_{i} \theta_{j}\right)$ (from 0 to 1 ) and another that represented competitive differences $\left.\left|\tilde{k}_{i}-\tilde{k}_{j}\right|\right)$. Subsequent analyses of species similarity were based on these two species distance and not on $\tilde{k}_{i}$ s and $\theta_{i} \mathrm{~s}$ directly. So, species competitive ability determines competitive exclusion in the lack of niche differences, while species versatility determines the niche overlap among species.

We simulated values for parameters $k_{i}$ and $\theta_{i}$ using the beta distribution, which is a continuous probability distribution defined on $[0,1]$ with two positive parameters: $\alpha$ and $\beta$. We used $\alpha$ and $\beta$ to control the means of $k_{i}$ and $\theta_{i}$ values and their variation. It is important to stress that low $\alpha$ values for $\theta_{i}$ means that, overall, species are highly versatile. Controlling the $\alpha$ and $\beta$ parameters in the beta distribution allowed us to simulate various scenarios for the distribution of fitness and niche differences in the species pool (Fig. 2). Differences 
(a) $\tilde{k} \sim \operatorname{beta}(1,5)$
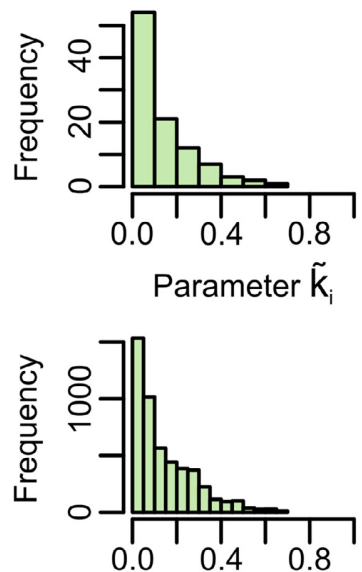

Fitness-based distances (b) $\tilde{k} \sim \operatorname{beta}(0.5,0.5)$
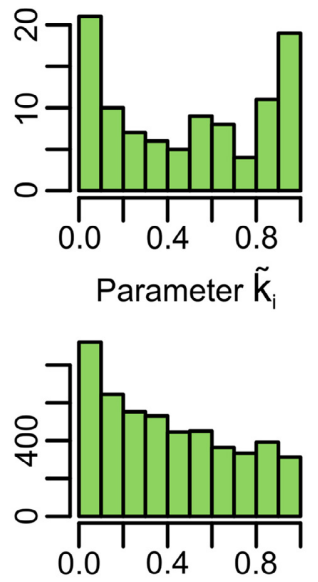

Fitness-based distances (c) $\tilde{\mathrm{k}} \sim \operatorname{beta}(2,2)$

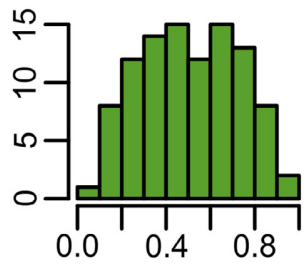

Parameter $\tilde{\mathrm{k}}_{\mathrm{i}}$

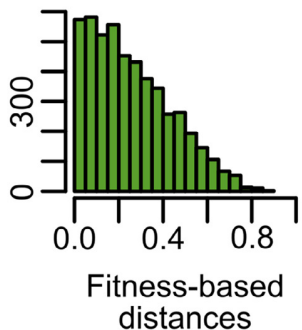

(d) $\tilde{k} \sim \operatorname{beta}(5,1)$

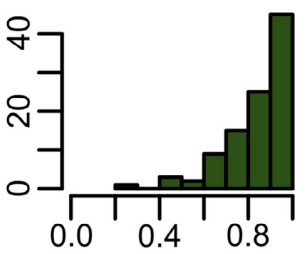

Parameter $\tilde{\mathrm{k}}_{\mathrm{i}}$

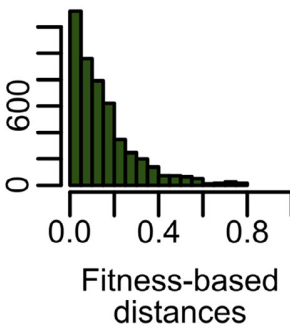

(e) $\theta \sim \operatorname{beta}(10,1)$
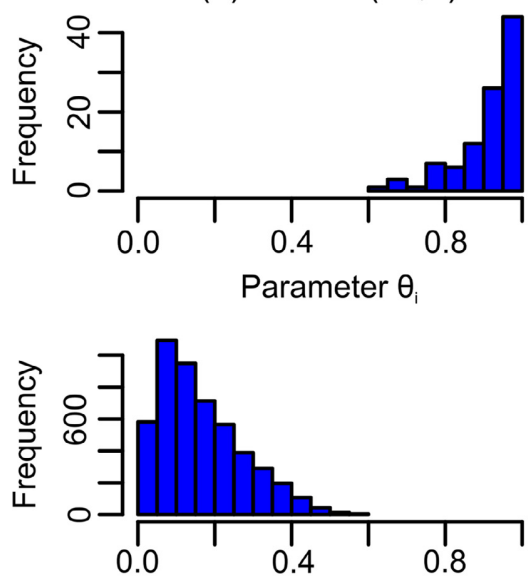

Niche-based distances (f) $\theta \sim \operatorname{beta}(10,5)$
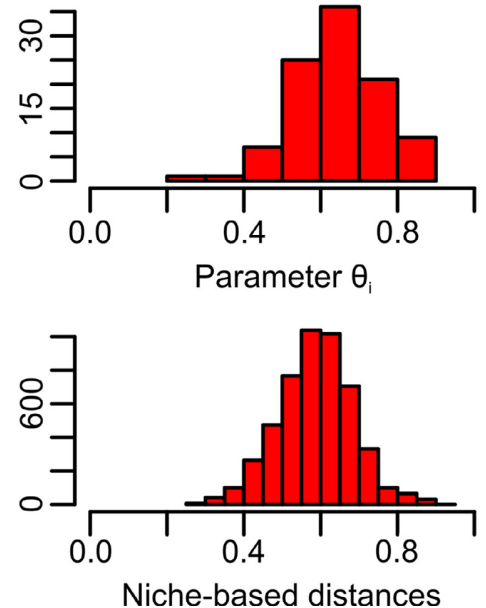

(g) $\theta \sim \operatorname{beta}(1,1)$
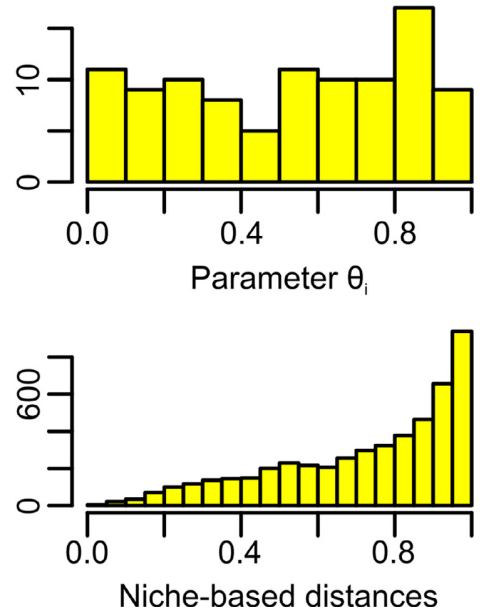

FIG. 2. Typical distributions used for $k$ and $\theta$ parameters using beta distributions. The $k$ s relate to species fitness and $\theta$ s relate to species interaction strength. Using the simulated species we calculated fitness-based distances between any two species $i$ and $j$ as and niche-based distances as $1-\theta_{i} \theta_{j}$. (a) A positive skewed distribution of $k \mathrm{~s}$ with most species having low fitness. (b) A U-shaped distribution of $k \mathrm{~s}$ with most species having low or high fitness and few species having medium fitness. (c) A for bell-shaped distribution of $k \mathrm{~s}$ with most species having medium fitness. (d) A negative skewed distribution of $k$ s with most species having high fitness. (e) A negative skewed distribution of $\theta_{i}$ s resulting in most species having low niche differences between them. (f) A bell-shaped distributions of $\theta_{i}$ s resulting in most species having medium niche differences between them. (g) A uniform distribution of $\theta_{i}$ s resulting in most of species having high niche differences between them.

between the parameters of the beta distribution (Fig. 2) are due to differences in the way fitness and interaction strength interfere in the model, fitness in an additive manner and interaction strength in a multiplicative manner.

Once the species pool was defined, we built the model to simulate community assembly. The model requires a given a priori number of colonization events to occupy a community. The species pool is unchanged so that a single species can try to invade a community several times. The first species is randomly selected from the pool and occupies a community. Then, the model randomly selects a second species to invade the community and applies the coexistence condition (Eq. 9) to decide whether the first and the second species can coexist in that community. If these species can coexist, the next (third) invader would be tested using the multispecies coexistence condition (Eq. 9). Subsequent colonizing species are tested for the coexistence condition with all species that already coexist there. The process of assembly ends when all the colonization events defined a priori were done. We also simulated scenarios where species have different colonization probabilities depending on their $k_{i}$ and $\theta_{i}$, one with a colonization-competition trade-off and another where species that rely mostly on a common resource have higher chance to colonize new patches. However, these simulations provided highly similar results to the random dispersal approach. Thus, these are only described in Appendix S3.

In this model, when the coexistence condition was not satisfied and the long-term growth rate of the invader was negative, then the invader was excluded. If the coexistence was not satisfied and the long-term growth rate of the invader was positive, then the species with the minimum long-term growth rate was excluded and the coexistence condition was 
tested in the new resulting community. This process was repeated until the coexistence condition was satisfied. We relied on previous work (Tregonning and Roberts 1979, Wilson and Lundberg 2004) to assume that the iterative removal of species with negative growth rates provides a reasonable approximation of the equilibrium community.

We defined a simulation as each round starting from simulating the species pool to the assembling of 100 communities. So, each simulation has one singular species pool to assemble 100 communities. A simulation thus ends with a set of 100 communities assembled under the same conditions.

\section{Community metrics}

To analyze the communities, we calculated species richness, beta diversity (Jaccard dissimilarity) and two metrics of species dispersion. Among species dispersion metrics, we used the standardized effect size of mean pairwise distances (SES.MPD) and that of the mean distance to the most similar species within communities (SES.MNTD, Webb 2000). We used the 'taxa shuffle' null model that randomizes 1,000 times the rows and columns of the species distance matrix. An additional metric of functional dispersion was also calculated. However this metric provided very similar results from SES.MNTD and SES.MPD, so we included these results in Appendix S3.

As a first step, we ran 100 simulations, with 100 invasions in each community, for each combination of fitness and niche distributions (Fig. 2). In these simulations, we first applied the species dispersion metrics (SES.MNTD and SES.MPD) to each assembled community, but separately to fitness distances and niche distances. Next, we applied the dispersion metrics to a combined niche and fitness-based distance between species named 'overall species distance' and defined as: $\frac{1}{2} \frac{\text { Fitness distance }}{\max (\text { Fitness distances })}+\frac{1}{2} \frac{\text { Niche distance }}{\max (\text { Niche distances) }}$, where maxima are over all species pairs in the species pool. Finally, to simulate studies gathering traits more related to niche or fitness differences among species (see Kraft et al. 2015), we modified the way we defined overall species distances as: $\frac{a}{a+b} \frac{\text { Fitness distance }}{\max (\text { Fitness distances })}+\frac{b}{a+b} \frac{\text { Niche distance }}{\text { max(Niche distances) }}$, where maxima also are over all species pairs in the regional species pool and $a$ and $b$ are positive parameters. This simulation represents studies gathering different number of traits related to niche differences (larger values of $b$ ) and fitness differences (larger values of $a$ ), or also simulates studies where one singular trait relates to both niche and fitness differences but with different weights (e.g., Godoy et al. 2014, Kraft et al. 2015). In a second step, we varied the number of colonization events per community considering 30, 50, 100, 300 and then 500 colonization events.

\section{RESULTS}

When communities were assembled by 100 colonization events, we found a positive trend in species richness by increasing the niche differences (and versatility) and the fitness values at the species pool scale (Fig. 3a). Beta diversity was high on average, independently of niche differences and species fitness at the species pool scale (Fig. 3b).

Considering the species dispersion metrics, when species distances were calculated using niche differences only, we found consistent overdispersion (Fig. 3c, d). With larger niche differences and higher fitness at the species pool scale, overdispersion tended to be more evident. When species distances were calculated using fitness differences only, we found clustering for most simulations (Fig. 3e, f). The exceptions were a tendency to randomness when mean species $k$ were low at the species pool scale (Fig 3c first boxplots within each color) and when niche differences were high at the species pool scale for the SES.MNTD metric (Fig. 3e, yellow boxplots). When overall species distances were calculated, we found a strong tendency towards overdispersion using the SES.MNTD metric (Fig. 3g), especially when niche differences at the species pool scale were large (species where highly versatile) (Fig 3g yellow boxes). The only exception happened when the species pool had small $k$ s values (Fig. $3 \mathrm{~g}$, first blue box).

When using the SES.MPD metric, results were more variable: (1) overdispersion when species had large niche differences at the species pool (Fig. 3h, yellow boxes) or similarly high $k$ s at the species pool (Fig. 3h, third boxes within each color); (2) a tendency to clustering when species $k \mathrm{~s}$ had a Ushaped distribution (Fig. 2h, forth blue and red boxes); (3) random patterns when species had low or medium $k$ and/or niche differences at the species pool (Fig. 3h, first and second blue and red boxes).

When varying the weights of niche differences and fitness differences in species overall distance (i.e., varying the parameters $a$ and $b$ when calculating overall distances), we found that the weights strongly impacted dispersion metrics, independently of the species pool properties. For brevity, we only show the results when species have medium niche differences and medium $k$ s values at the species pool scale. We found that SES.MNTD was overdispersed even when we gave more weight to fitness differences than niche differences (Fig. 4a). There was a tendency towards randomness and clustering when fitness differences were four to five times more important than niche differences (Fig. 4a). Using SES.MPD, we found random patterns when the weights of niche and fitness differences was the same, but clustering and overdispersion when there were more weights to fitness and niche differences, respectively (Fig. 4b).

Varying the number of sequential colonization events led to the same general conclusions (Fig .5). For brevity, we only show the results obtained with medium niche differences and fitness values at the species pool scale. Community richness increased with more colonization events, but stabilized after some point (Fig. 5a). Beta diversity decreased with increasing colonization reaching very low levels after 300 and 500 colonization events (Fig. 5b). We found consistent niche overdispersion and fitness clustering (Fig. 5c-f). Using overall species distance we found overdispersion using SES.MNTD with a minimum of 50 colonization events and only randomness using SES.MPD (Fig. 5g, h). Overall, dispersion patterns became more evident with increasing number of colonization events.

\section{Discussion}

Community assembly studies have the ambitious aim of inferring processes by analyzing similarities in traits or in evolutionary history among species in a given community 

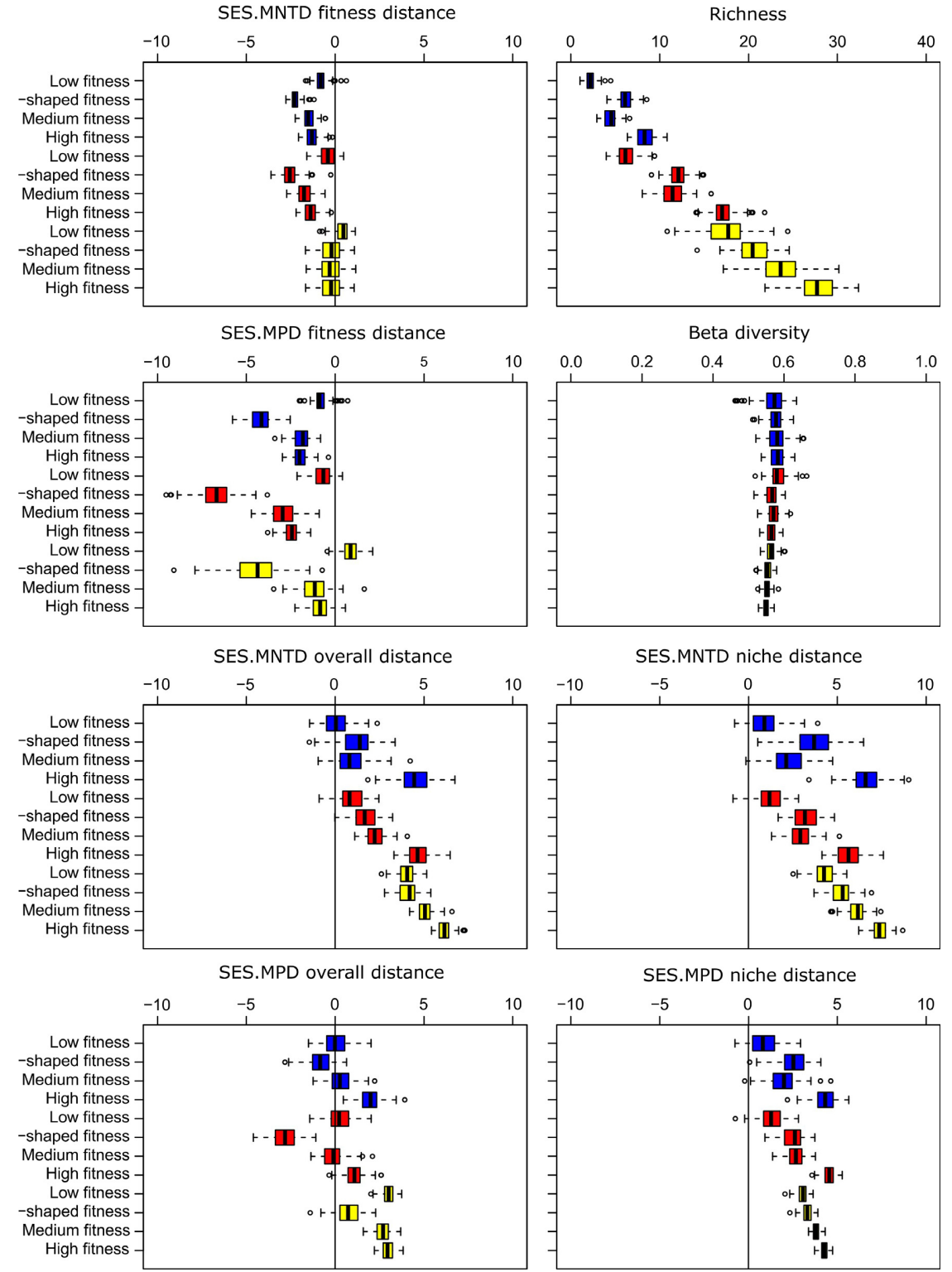

FIG. 3. Variation in species richness, Jaccard dissimilarity and species dispersion metrics when simulating community assembly using different species pools. Blue boxplots are results for species pools with average low niche differences, red boxplots for medium niche differences and yellow for high niche differences. Within each color, the first boxplot is when species have low fitness at the species pool scale, the second when species have medium fitness values, the third when species have high fitness values and the forth when species have a U-shaped distribution. We first applied indices SES-MNTD and SES-MPD separately to niche distances and fitness distances between species and then to the overall functional distances which combines niche and fitness as defined in the main text.

(Weiher and Keddy 1995, Webb 2000). We show, however, that such approaches can suffer from two types of Achilles' heels when linking patterns to processes. The first is when different processes result in the same pattern. We found that communities containing very similar species - a pattern used to infer environmental filtering (Weiher and Keddy 1995, Webb 2000) - can also be the result of competition alone (Mayfield and Levine 2010). The second is when one process leads to different patterns. We found that competition can result in communities containing very distinct species (i.e., an overdispersed pattern, as predicted by the traditional framework), but also in communities with clustered (as predicted by Mayfield and Levine 2010) and even random structure.
These problems likely arise because we simulated communities with the inclusion of two scenarios rarely considered in previous studies: simultaneous competition among multiple competitors (e.g., Godoy et al. 2017) and species with different levels of versatility to exploit marginal resources. These results bring concerns to the use of the traditional community assembly approach, but enabled us to do a step forward in understanding recurrent patterns of communities in nature.

Here, we introduced an extension of the pairwise theory of coexistence using a variation of the MacArthur's consumer-resource model (Chesson 1990) that considers species versatility to determine the interaction strength among species. This new model differs in important points from the 

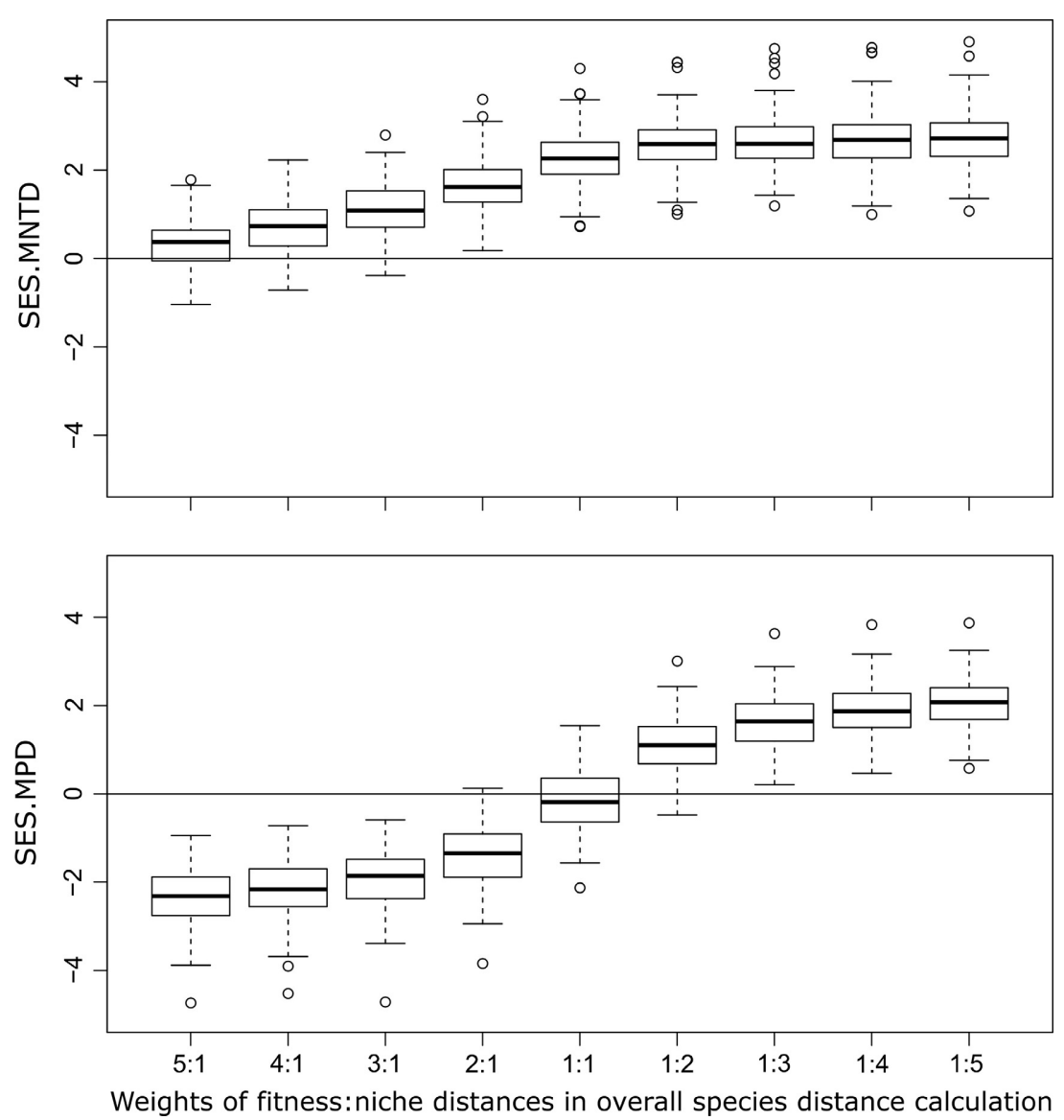

FIG. 4. Result of species dispersion metrics when species distance is calculated using different weights for niche and fitness differences. Community assembly was performed using a species pool with medium niche differences and medium fitness values at the species pool scale.

traditional view of competition (Fig. 1) but yield concordant patterns with previous inferences on the impacts of ecological versatility in community assembly. For example, species richness increased with species versatility in the community and in the species pool scale (Bellwood et al. 2006, Belmaker et al. 2012). Additionally, we found that versatility provides important niche differences and promotes coexistence when competitors from the pool are very strong (high fitness), as already suggested for empirical communities (e.g., cichlids, Liem 1980). Overall, these concordances from theory and data emphasize the important role that versatility may play in the assembly of natural communities.

Our findings indicate that empirical studies able to separate niche from fitness traits (e.g., Kraft et al. 2015) could have found species overdispersion if species distances reflected niche differences, but clustering if it reflected fitness differences. However, in the case traits impact both fitness and niche differences (Adler et al. 2013, Kraft et al. 2015), our results suggest that MPD metrics can also be random. Previous empirical studies indeed found clustering and overdispersion using different traits with an overall species clustering, but the interpretation relied on the opposing effects of environmental filtering and limiting similarity (e.g., Swenson and Enquist 2009). Here, we demonstrated how these same patterns can arise due to competition only, when fitness have a U-shaped distribution (Fig. 3h). The Ushaped distribution is the only bimodal distribution we used and it demonstrated to promote different patterns compared to when fitness has unimodal distributions. Empirically, traits with bimodal distribution are recurrent in nature (e.g., marine zooplankton and stream biotas with bimodal size distribution Warwick and Clarke 1984, Poff et al. 1993). So, as these patterns can be found in nature, it is likely that clustering in some traits (related to competitive ability) and overdispersion in others (related to species versatility) are also possible when competition rules the assembling.

The distributions of niche and fitness differences in the species pool had pivotal effects in determining the level of species dispersion, especially in SES.MPD. With large niche differences in the species pool (i.e., species are highly versatile), overdispersion emerged consistently when using overall species distances. This is likely the result of a possible coexistence when most species have large niche differences, independently of their fitness differences (Chu and Adler 2015). A strong tendency towards overall overdispersion also emerged with high fitness at the species pool scale. This contradicts the idea that communities dominated by strong competitors should have clustered patterns (Mayfield and Levine 2010), at least when species vary in their dependency for a common resource. Actually we found that, in these cases, species would tend to be high versatile to overcome possible large fitness disadvantages. In a more conservative case, when the species pool has medium fitness values and medium niche differences, 

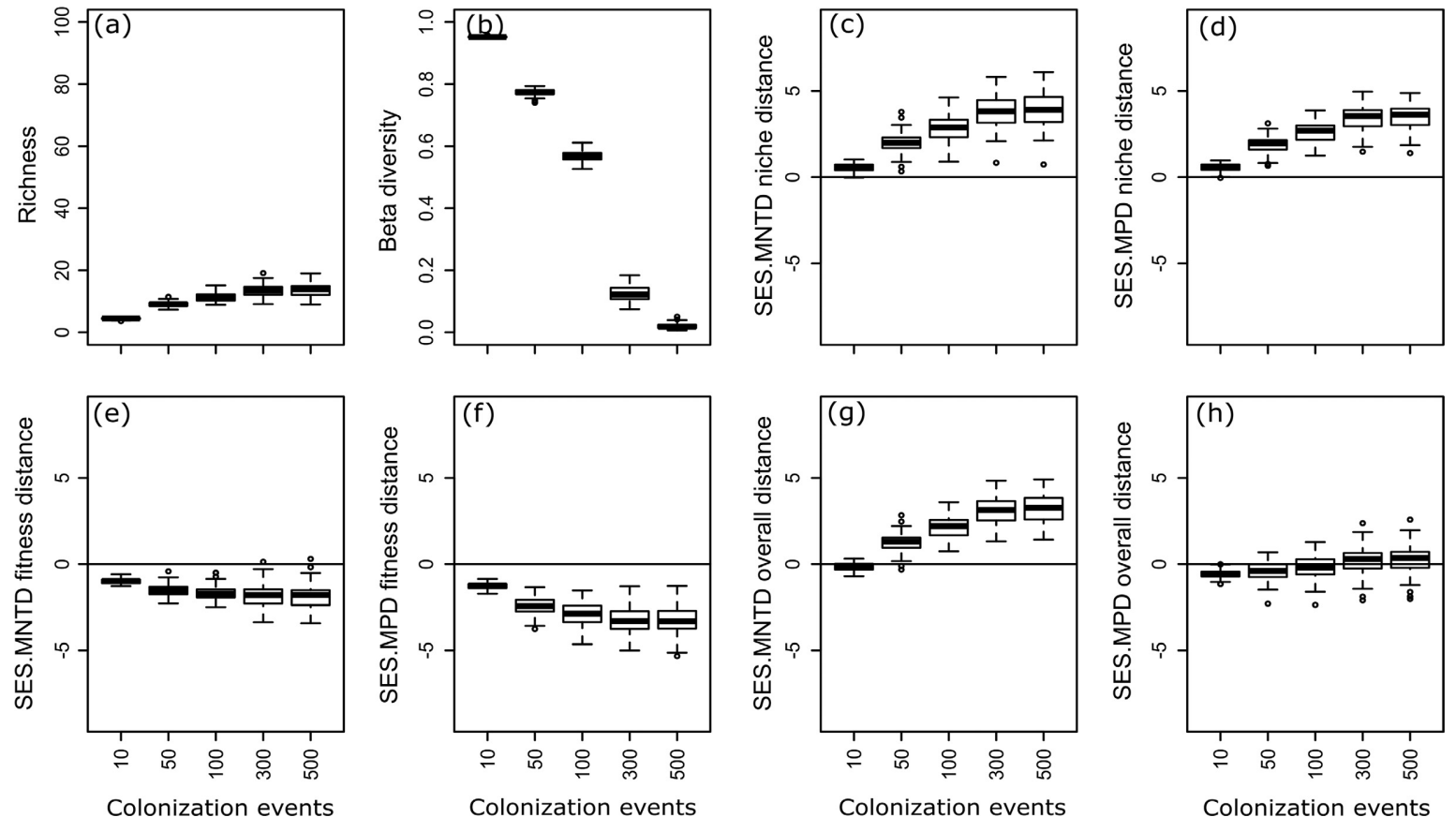

FIG. 5. Results of richness, Jaccard dissimilarity and species dispersion metrics when we varied the number of sequential colonization events during community assembly. Community assembly was performed using a species pool with medium niche differences and fitness values at the species pool scale. We first applied indices SES-MNTD and SES-MPD separately to niche distances and fitness distances between species and then to the overall functional distances which combines niche and fitness as defined in the main text.

overall distance was in most cases random (Figs. $2 \mathrm{~h}$ and 4h). Given the commonness of a bell-shaped distribution of traits in nature (e.g., traits evolving under a Brownian motion commonly result in normal distributions around an ancestral trait value, Kraft et al. 2007), it is not surprising that most studies to date have observed random patterns when analyzing mean distance-based metrics $(82 \%$ according to a review by Götzenberger et al. 2012). Thus, we support recent studies cautioning the inference of stochastic processes, e.g., random variation in birth and death rates, as the main processes governing communities with random dispersion patterns (Gallien 2017).

Besides the cautionary notes, we found one consistent result across most model variations: consistent overdispersion when using the SES.MNTD metric. This finding supports the importance of minimum niche differences between species in stable multispecies communities (Godoy et al. 2017). This is likely because a high ability to escape competition for a common resource will help overshadow the competitive differences with all other species (e.g., Liem 1980). After years of criticism (Mayfield and Levine 2010, Adler et al. 2013, Gallien 2017), we add new evidence that the signature of pairwise niche differences in communities assembled by multispecies competition is the same overdispersion hypothesized by Weiher and Keddy (1995), even when indirect effects due to multiple interactions could act to weaken such pattern (Godoy et al. 2017). It is also important to note that Godoy et al. (2017) used a different model of competition (an annual plant species model, Godoy et al. 2014), but also found the similar pattern of overdispersion. This highlights the general importance of minimum niche differences even when considering competition in different ways.
In this recent study, Godoy et al. (2017) also discussed the role of niche differences and intransitivity (rock-paper-scissor dynamic) in maintaining multispecies communities, explaining that both would give similar empirical signals of overdispersion. However, for intransitive competition to promote coexistence, species need to differ in their ability to compete for different resources, thus making possible that a superior competitor for resource $\mathrm{A}$ is worse when competing for resource B (Gallien et al. 2017). However, this scenario cannot arise in our model as competitive ability is a general characteristic, not related to each specific resource; i.e., we would never have a species with $k=10$ being outcompeted by a species with $k=5$. In our model, the coexistence of species with larger differences than expected at random is not related to possible intransitivity but specifically to stabilizing versatility in resources used. So, by using a model that isolates the role of niche differences, we are able to support the idea that intransitivity emerging from multispecies competition should not be pivotal for species coexistence in the absence of sufficient pairwise niche differences (Godoy et al. 2017).

Finally, we must punctuate some limits of our approach. First, our model is an interaction chain, where a species A has an indirect effect on species $\mathrm{C}$ by also competing with the common competitor B. Another type of multispecies competition considers higher order interactions, where pairwise competition changes in the presence of a third competitor, so that the impact of $\mathrm{A}$ on $\mathrm{B}$ is different when in pairwise or in triplets. The distinction between interaction chains and higher-order interactions is determined by whether the indirect effect emerges from a change in competitor density (interaction chain) or a change in the per capita competitive effects (higher-order interaction). As we 
did not consider such dynamics in our model, we cannot anticipate the impacts that these would have on the patterns we found (see Levine et al. 2017). Second, in our study, we did not explicitly include temporal or spatial variation mechanisms of coexistence. With more varying niche dimensions, it is possible that local differences in resource use became less important and thus local overdispersion may become weaker (Kraft et al. 2015). Third, our model is strongly deterministic, not including neutral population dynamics that could modify community patterns. Studies on these dynamics have shown that removing niche differences can have strong impacts on species coexistence. For example, D'Andrea and Ostling (2017) found that neutral cases can even harbor larger number of coexisting species than niche based scenarios, especially if the number of available niches is limited. So, a way forward would be to investigate how such dynamics would modify the patterns found here.

Concluding, here we introduced a new model of competition where species versatility promotes niche differences. This model proved to be an interesting variation of the MacArthur's consumer-resource model when species vary in their dependency for a commonly exploited resource. Competition in nature is complex and should happen, not only in both ways, but also in many other ways. Besides this high complexity, we found that overdispersion using nearest neighbor metrics was a recurrent pattern and thus could signalize a master role that competition plays during community assembly. After some years of debate, our model and results highlight the role of species versatility and niche differences and bring new perspectives for those aiming to infer the role of competition in molding community patterns.

\section{ACKNOWLEDGMENTS}

We are thankful to Raul Costa Pereira, Andros Gianuca, Franck Jabot, Ian Carroll, François Massol, Matthew Leibold and Peter Chesson for comments that sharpened our ideas. We also thank Annette Ostling, Nathan Kraft and an anonymous referee for comments and discussions during the review process that greatly improved our manuscript. This study was funded by grants \#2013/ 50424-1, \#2013/20540-0, \#2014/24532-4 São Paulo Research Foundation (FAPESP) and by grant 403723/2012-4, Conselho Nacional de Desenvolvimento Científico e Tecnológico (CNPq). VSS thanks the Centre d'Ecologie et de Sciences de la Conservation (CESCO) from the Muséum National d'Histoire Naturelle in Paris for kindly receiving him during part of the elaboration of this manuscript.

\section{Literature Cited}

Adler, P. B., A. Fajardo, A. R. Kleinhesselink, and N. J. B. Kraft. 2013. Trait-based tests of coexistence mechanisms. Ecology Letters 16:1294-1306.

Bellwood, D. R., P. C. Wainwright, C. J. Fulton, and A. S. Hoey. 2006. Functional versatility supports coral reef biodiversity. Proceedings of the Royal Society of London B: Biological Sciences 273:101-107.

Belmaker, J., C. H. Sekercioglu, and W. Jetz. 2012. Global patterns of specialization and coexistence in bird assemblages. Journal of Biogeography 39:193-203.

Cadotte, M. W., and C. M. Tucker. 2017. Should environmental filtering be abandoned? Trends in Ecology and Evolution 32:429-437.

Chesson, P. 1990. MacArthur's consumer-resource model. Theoretical Population Biology 37:26-38.

Chesson, P. 1991. A need for niches? Trends in Ecology and Evolution 6:26-28.
Chesson, P. 2000. Mechanisms of maintenance of species diversity. Annual Review of Ecology, Evolution and Systematics 31: 343-358.

Chu, C., and P. B. Adler. 2015. Large niche differences emerge at the recruitment stage to stabilize grassland coexistence. Ecological Monographs 85:373-392.

D'Andrea, R., and A. Ostling. 2016. Challenges in linking trait patterns to niche differentiation. Oikos 125:1369-1385.

D'Andrea, R., and A. Ostling. 2017. Biodiversity maintenance may be lower under partial niche differentiation than under neutrality. Ecology 98:3211-3218.

Gallien, L. 2017. Intransitive competition and its effects on community functional diversity. Oikos 126:615-623.

Gallien, L., N. E. Zimmermann, J. M. Levine, and P. B. Adler. 2017. The effects of intransitive competition on coexistence. Ecology Letters 20:791-800.

Godoy, O., N. J. B. Kraft, and J. M. Levine. 2014. Phylogenetic relatedness and the determinants of competitive outcomes. Ecology Letters 17:836-844.

Godoy, O., D. B. Stouffer, N. J. Kraft, and J. M. Levine. 2017. Intransitivity is infrequent and fails to promote annual plant coexistence without pairwise niche differences. Ecology 98:1193-1200.

Götzenberger, L., et al. 2012. Ecological assembly rules in plant communities-approaches, patterns and prospects. Biological Reviews 87:111-127.

Herben, T., and D. E. Goldberg. 2014. Community assembly by limiting similarity vs. competitive hierarchies: testing the consequences of dispersion of individual traits. Journal of Ecology 102:156-166.

HilleRisLambers, J., P. B. Adler, W. S. Harpole, J. M. Levine, and M. M. Mayfield. 2012. Rethinking community assembly through the lens of coexistence theory. Annual Review of Ecology, Evolution and Systematics 43:227-248.

Kraft, N. J. B., W. K. Cornwell, C. O. Webb, and D. D. Ackerly. 2007. Trait evolution, community assembly, and the phylogenetic structure of ecological communities. American Naturalist 170:271-283.

Kraft, N. J. B., O. Godoy, and J. M. Levine. 2015. Plant functional traits and the multidimensional nature of species coexistence. Proceedings of the National Academy of Sciences 112:797-802.

Lawlor, L. R. 1979. Direct and indirect effects of n-species competition. Oecologia 43:355-364.

Levine, S. H. 1976. Competitive interactions in ecosystems. American Naturalist 110:903-910.

Levine, J. M., J. Bascompte, P. B. Adler, and S. Allesina. 2017. Beyond pairwise mechanisms of species coexistence in complex communities. Nature 546:56-64.

Liem, K. F. 1980. Adaptive significance of intra-and interspecific differences in the feeding repertoires of cichlid fishes. American Zoologist 20:295-314.

Liem, K. F. 1990. Aquatic versus terrestrial feeding modes: possible impacts on the trophic ecology of vertebrates. American Zoologist 30:209-221.

MacArthur, R. 1970. Species packing and competitive equilibrium for many species. Theoretical Population Biology 1:1-11.

MacArthur, R. H., and R. Levins. 1967. The limiting similarity, convergence, and divergence of coexisting species. American Naturalist 101:377-385.

Mayfield, M. M., and J. M. Levine. 2010. Opposing effects of competitive exclusion on the phylogenetic structure of communities. Ecology Letters 13:1085-1093.

Pianka, E. R. 1974. Niche overlap and diffuse competition. Proceedings of the National Academy of Sciences 71:2141-2145.

Poff, N. L., M. A. Palmer, P. L. Angermeier, R. L. Vadas, C. C. Hakenkamp, A. Bely, P. Arensburger, and A. P. Martin. 1993. Size structure of the metazoan community in a Piedmont stream. Oecologia 95:202-209.

Swenson, N. G., and B. J. Enquist. 2009. Opposing assembly mechanisms in a Neotropical dry forest: implications for phylogenetic and functional community ecology. Ecology 90:2161-2170.

Tregonning, K., and A. Roberts. 1979. Complex systems which evolve towards homeostatis. Nature 281:563-564. 
Warwick, R. M., and K. R. Clarke. 1984. Species size distributions in marine benthic communities. Oecologia 61:32-41.

Webb, C. O. 2000. Exploring the phylogenetic structure of ecological communities: an example for rain forest trees. American Naturalist $156: 145-155$.
Weiher, E., and P. A. Keddy. 1995. Assembly rules, null models, and trait dispersion: new questions from old patterns. Oikos 74:159-164. Wilson, W. G., and P. Lundberg. 2004. Biodiversity and the LotkaVolterra theory of species interactions: open systems and the distribution of logarithmic densities. Proceedings of the Royal Society of London B: Biological Sciences 271:1977-1984.

\section{SUPPORTING INFORMATION}

Additional supporting information may be found in the online version of this article at http://onlinelibrary.wiley.com/doi/10.1002/ecy. 2197/suppinfo 